\title{
Epithelial Modelling Platform: A Tool for Model Discovery and Assembly with the Physiome Model Repository
}

\author{
Dewan M. Sarwar, Yuda Munarko and David P. Nickerson \\ Auckland Bioengineering Institute, University of Auckland, Auckland, New Zealand
}

\begin{abstract}
In this paper we present a web-based platform enabling scientists to construct a novel epithelial transport model to investigate their hypotheses, aided by building on existing models discovered in the Physiome Model Repository (PMR). We have comprehensively annotated a cohort of epithelial transport models deposited in the PMR as a seeding collection of building blocks that are freely available for reuse. On the platform, users are able to semantically display models for visualization, graphical editing, and model assembly. In addition, we leverage web services from the European Bioinformatics Institute (EBI) to help rank similar models based on the suggestions provided by the platform. In addition to potential use in biomedical and clinical research, novice modellers could use our platform as a learning tool. The source code and links to a live demonstration of the platform are available at https://github.com/dewancse/ epithelial-modelling-platform.
\end{abstract}

Keywords: CellML, Physiome Model Repository, Semantic annotation, Model discovery, Model assembly, Epithelial transport

\section{INTRODUCTION}

The IUPS Physiome Project (Hunter and Borg, 2003) and the Virtual Physiological Human (VPH) (Hunter et al., 2010) have introduced tools and open source software for scientists to conduct biomedical research (Garny et al., 2010; Cooper et al., 2010). For example, biomedical engineers and clinicians often utilize computational models to investigate experimental or clinical hypotheses that are difficult or expensive to achieve experimentally using animal or human subjects. Modern tools and technologies help them to quickly and precisely test such hypotheses which may involve disparate biophysical symptoms and/or mechanisms such as clinical observations, diseases, and drug actions through physiology pathways (de Bono et al. 2016).

In this paper, we present a web-based platform, the Epithelial Modelling Platform (EMP), which will enable biomedical engineers and clinicians to discover epithelial transport models with a view to constructing a new epithelial model. This new model will help them to investigate specific research questions and hypotheses. To prepare a model discovery environment, mathematics- and physics-based epithelial transport models are integrated with biological information. Integration of such information in the context of biology is called model annotation. Model annotation captures semantic meanings of biological phenomena encapsulated in mathematics and thus hides underlying mathematical equations (Lister et al., 2009). In order to represent biological phenomena, model annotation makes use of a rich set of controlled vocabulary, i.e. ontologies (Courtot et al., 2011). This often requires multiple reference ontologies to represent such phenomena, which is called composite annotation (Gennari et al., 2011). Model annotation is a time-consuming process and thus requires automated process. For this, SemGen has been developed to annotate biosimulation models (Neal et al., 2018). We have deposited the annotated 
information and the models in the Physiome Model Repository (PMR) (Yu et al., 2011) where information is discoverable, accessible, and persistent. PMR follows FAIR (Wilkinson and et al., 2016) principles; i.e. information in PMR is Findable, Accessible, Interoperable, and Reusable (FAIR).

Prior research in (Galdzicki et al., 2011; Neal et al., 2013) demonstrates that model discovery can be performed by utilizing semantic querying technologies. Similarly, with the provision of modern technologies, knowledge discovery has introduced a range of tools and open source software such as DisGeNET (Piñero et al., 2015), ApiNATOMY (de Bono et al., 2014), JWS Online (Wimalaratne et al., 2004), Saint (Lister et al., 2009), semanticSBML (Krause et al., 2010), eSolve (de Boer et al., 2017), Chalkboard (Cook et al., 2007), Physiomaps (Cook et al., 2013). A recent initiative in (Cooper et al., 2015a), the Cardiac Electrophysiology Web Lab, has introduced an online simulation environment to compare simulation experiments. Such virtual experiments are analogous to in silico environment of in vivo and in vitro experiments, or wet lab experiments (Cooper et al., 2015b).

In addition to model discovery, we have assembled the discovered models on the platform with a view to constructing a new epithelial model. Model construction integrates components from various source models. SemGen can compose models in CellML (Cuellar et al., 2003) and SBML (Hucka et al., 2003) format. CellML and SBML are XML-based languages to describe biological processes. Specifically, SemGen internally converts models into OWL format and then does model composition (Hoehndorf et al., 2011). In contrast, we have composed models by utilizing libCellML (libCellML, 2019). libCellML is a library to serialize, validate, and instantiate a CellML model. A great deal of previous research on model composition is investigated in (Neal et al., 2015; Gennari et al., 2008; Neal et al., 2009; Krause et al., 2010; Coskun et al., 2013).

Here we present a model discovery and assembly approach which is a new contribution to PMR. In addition to potential use in biomedical and clinical research, novice modellers could use our platform as a learning tool. This platform would demonstrate how semantic web technologies and methodologies can contribute to educational purpose. In the section Materials and Methods, we present our current workflow of the EMP, the standards and software tools we have used to encode the computational models and semantic annotations for our exemplar cohort of models, and the technologies that enable the implementation of our platform. This platform, which makes use of the encoded models and semantic annotations to discover, explore, and visualize models, is discussed in the Results section. The discussion section summarizes the model discovery and assembly of the EMP with some concluding remarks.

\section{MATERIALS AND METHODS}

Fig. 1 presents an overview of the knowledge management platform being developed and utilized in this work. On the client side, content creation and editing applications like OpenCOR (Garny and Hunter, 2015) are used to encode computational models and associated information in computable formats. Such applications work on local workspaces, which can be synchronized with cloud-based repositories such as the PMR. Synchronization with PMR ensures persistent, resolvable, and discoverable identifiers for all resources in the workspace. For composite annotation, we used the SemGen tool and deposited this annotation as metadata in PMR (Sarwar et al. 2018). Web-based applications such as our EMP are then able to leverage the knowledge stored in PMR to discover models, and semantically assemble these models on the platform for visualization, graphical editing, and model assembly and construction.

Our EMP has become a key demonstrator application of the knowledge management system. The aim was to discover models from the PMR and assemble these on the platform with the provision of the EBI 


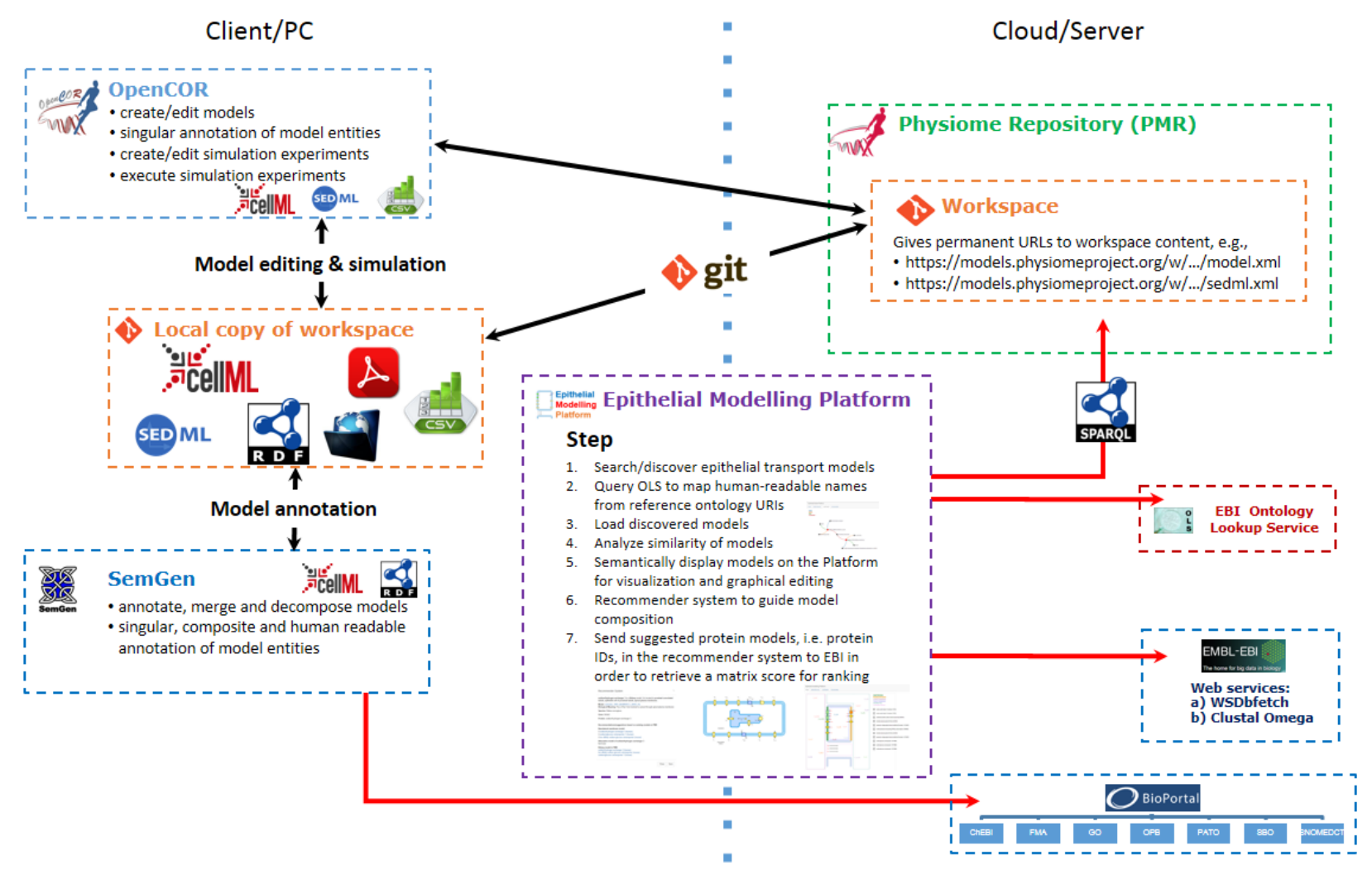

Figure 1. Illustrative example of current workflow for: 1) using OpenCOR to edit CellML models; 2) using SemGen to annotate the biological knowledge and deposit them in PMR; 3) using a git client to synchronize the local data to PMR; and 4) using our Epithelial Modelling Platform to discover CellML models and then semantically display these models on the platform for visualization, graphical editing, and model assembly and construction.

ontology lookup service (OLS) (OLS, 2019) and web services as shown in Fig. 1. The seven key steps in this process are described as follows. (1) Search/discover models of interest using the information extracted from the annotations deposited in PMR. (2) In this case, the EBI OLS is used to map human-readable names to reference ontology identifiers. (3) The user will then select models from the discovered model set and will make a shortlist of models for visualization and graphical editing on the platform. Similar to (2), the EBI OLS is used for name resolution. (4) In the shortlisted space, we have provided an option for the user to find similar components between a set of selected models, enabling users to identify overlap between models or points of integration. (5) We then semantically visualize the shortlisted models on a scalable vector graphics (SVG), which is an XML-based two-dimensional interactive display format, display panel for graphical editing. Specifically, the shortlisted models are placed on a specific membrane or compartment based on the annotation in PMR. (6) A recommender system is implemented to help the user enhance and extend the model they are building by providing links, for example, to models of similar transporters or transporters of the same solutes in different membrane locations. Such user-editing will automatically change the default settings of the models on the platform. Suggestions in this recommender system are provided from the available annotated apical or basolateral membrane models. (7) The EBI web services are used to rank the available models in the recommender system.

Computational tools and standards have been evolved over the years by The COmputational Modeling in BIology NEtwork (COMBINE) (COMBINE, 2019) and the World Wide Web Consortium (W3C) in order 
to collaborate and disseminate tools and standards. Some of the tools and standards used in our EMP are described in (Sarwar et al., 2018).

\section{RESULTS}

We have developed the EMP to discover and explore computational models of interest, as well as to semantically visualize the discovered models on the platform for graphical editing and model assembly with a view to constructing a new epithelial model. In particular, this involves annotation of mathematical models with biological information and generation of metadata using the SemGen tool so that our EMP could discover models from the metadata. Annotation of a cohort of epithelial transport models have been discussed in (Sarwar et al. 2018), therefore, following section presents a detailed workflow of the EMP.

\section{Discover CelIML Models}

The first workflow of the EMP is to discover and explore models of interest extracted from the annotation in PMR. Fig. 2 shows a list of discovered models for a search query "flux of sodium" from PMR. From this list, the user can investigate various options such as CellML model entity - name of the model, component name and variable name; biological meaning deposited in PMR; protein name; and species and genes used during experiments of the associated models. In addition, the user can navigate to the "view" option to explore more information about a model. The user can make a shortlist of models by navigating through the "Add to Model" option and can return here in order to rediscover models.

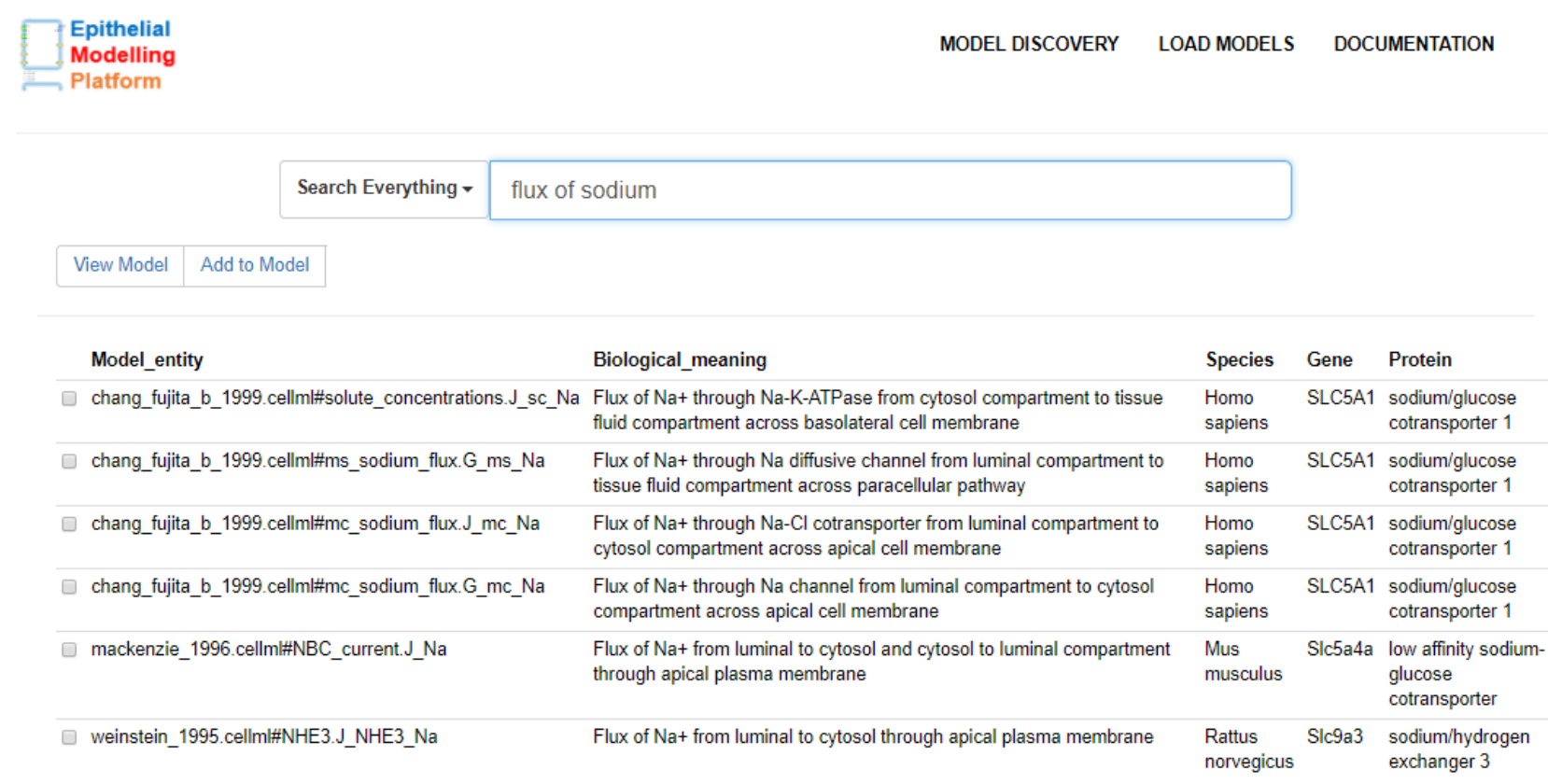

Figure 2. Model discovery interface to search for models in PMR that are relevant to the query "flux of sodium". By querying the annotations stored in PMR, this interface retrieves components from the NHE3 (Weinstein, 1995), SGLT2 (Mackenzie et al., 1996), and an epithelial cell model (Chang and Fujita, 1999).

Users are able to enter human-readable search terms and phrases or choose to enter specific vocabulary terms to search for. When discovering resources in PMR relevant to a given search phrase, we first decompose the English prose into a series of semantic queries which are executed on the PMR knowledge base and ranked according to how well the discovered resource matches the initial search query. Continuing 
our example, the phrase shown in Figure 2 will best match any resource associated directly with a sodium flux, but other sodium-related resources will also be returned with a lower ranking in the search results. To achieve that, we have maintained a static dictionary with key and value pairs to map searched text to reference ontology terms. For example, for a search query "flux of sodium", the model discovery interface will map flux and sodium to OPB and ChEBI ontology terms, respectively, as defined in the dictionary.

In addition to model discovery, users are able to add protein models by querying to the bioportal web service. This begins with entering a protein name in the "Add Model" interface of the EMP and then the bioportal web service returns a protein ID from the protein ontology (PR). Afterwards, the interface sends this protein ID to the OLS to retrieve species and gene names. Next, users enter the rate of concentration of solutes (i.e. flux) moving between compartments across apical or basolateral membrane. After filling in the required information, metadata will be generated and a CellML model will be serialized. In the future, we will implement a notification system in order to notify this new CellML model to the CellML editorial board so that they can fill in the required ODE-based equations and metadata of this new CellML model.

\section{Load Discovered Models}

As mentioned above, the user can make a shortlist of models from the discovered models for visualization, graphical editing, and model assembly and construction. Fig. 3 presents an example where the Biological_meaning in Fig. 22 is replaced with Compartment, and Located_in on the right of this example is added with a view to quickly provide more useful information to the user. In this space, we have provided some useful options: "view" option to get a detailed overview of a model; "delete" option to remove models if the user is not interested in considering these models on the platform; "visualization" option to find a similarity between a set of selected models; and "epithelial platform" option to navigate to the SVG-based interface and to semantically display the shortlisted models, which is discussed below.

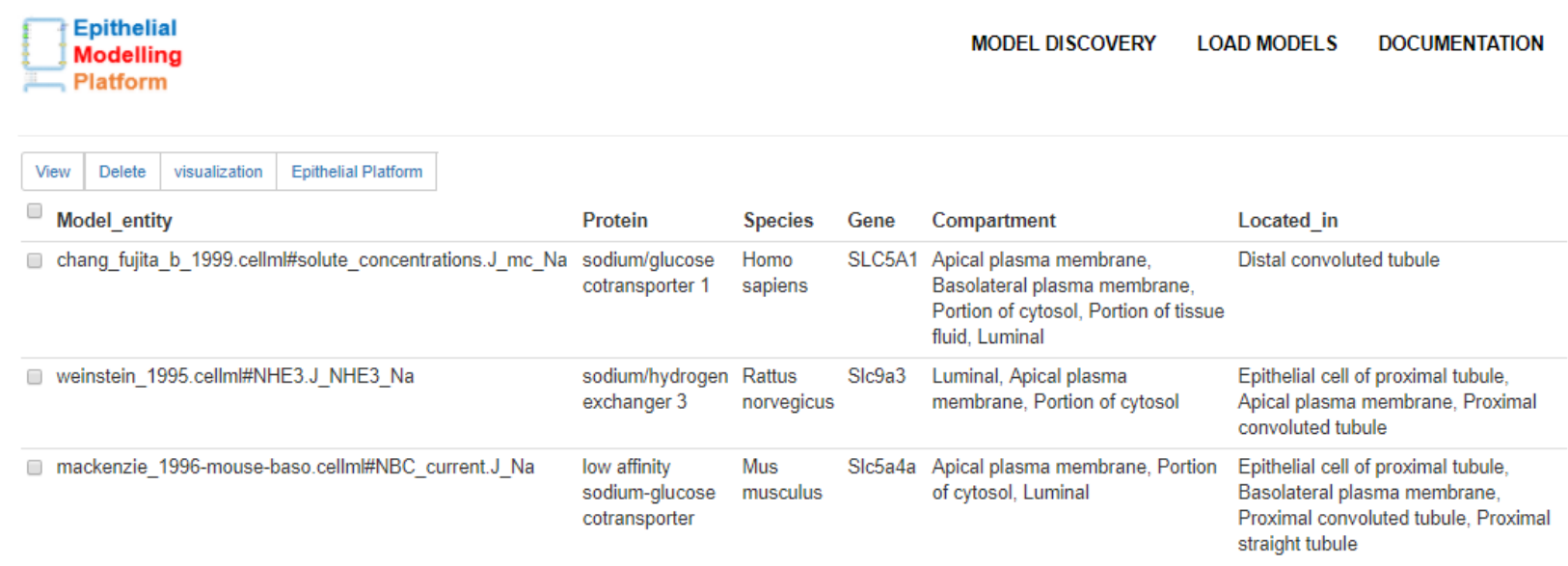

Figure 3. List of models for visualization and graphical editing on the platform. These models are selected from the model discovery interface illustrated in Fig. 2 .

\section{Models of Similarity}

Discovering similarity between models is vital to investigate coupling point(s), whereas visualization quickly conveys this investigation to the user. For this, we have developed models of similarity feature in the EMP. We aim to visually identify similar components, such as species, genes, and compartments between the selected models from the shortlist of models space. Fig. 4 shows a similar component between the 
NHE3 (Weinstein, 1995) and SGLT2 (Mackenzie et al., 1996) models. In this example, both models have same compartment. Nodes and edges are represented with unique colors to make the model distinguishable. Overall, by using this feature, the user would be able to analyze models and could choose relevant models in order to construct an epithelial model.

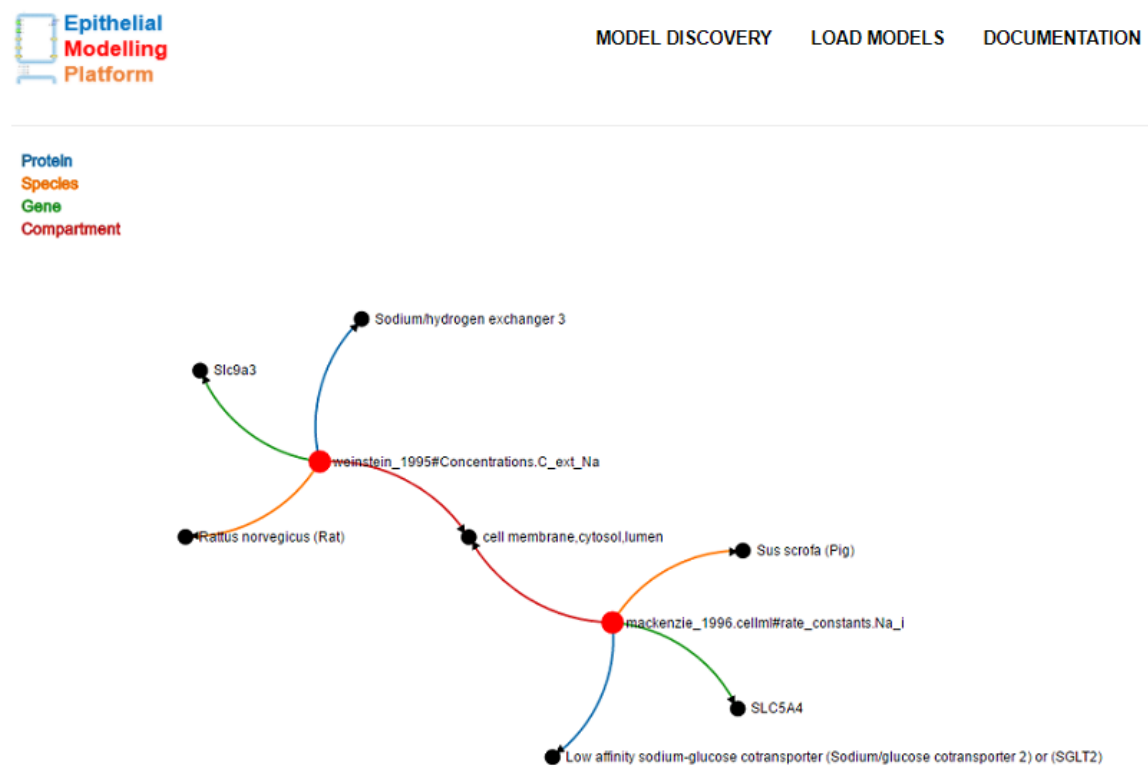

Figure 4. Illustrating the similarity between the NHE3 (Weinstein, 1995) and SGLT2 (Mackenzie et al., 1996) models. In this case, the transporter being modelled is located in the same compartment, labeled here with a unique colour. For convenience, we used "lumen" as a replacement of portion of renal filtrate in distal convoluted tubule and "cytosol" as a replacement of portion of cytosol in epithelial cell of distal tubule.

\section{Modelling Platform}

Fig. 5 presents an example of our SVG-based model visualization, graphical editing, and model assembly and construction platform. This platform has five compartments: portion of renal filtrate in distal convoluted tubule (luminal compartment), portion of cytosol in epithelial cell of distal tubule (cytosol compartment), paracellular, portion of tissue fluid in epithelial cell of distal tubule (interstitial fluid compartment), and blood capillary; and three membranes: apical, basolateral, and capillary. These compartments and membranes have been annotated with unique colors as shown in the top-right corner of this example. In order to distinguish between fluxes and channels, we have used various shapes, as described in Figure 5 .

The paracellular compartment separates two epithelial cells where diffusive fluxes are semantically placed on this compartment. In a similar manner, fluxes and channels are placed on a membrane based on the biological semantics. Before placing these fluxes and channels on a membrane, the platform checks its mediator (either apical, basolateral, or capillary membrane ID, and a protein ID) and shape (circles for fluxes or polygons for channels). A co-transporter, for example, $\mathrm{Na}-\mathrm{Cl}$ co-transporter (TSC) in Fig. 5 , is constructed if both $\left(\mathrm{Na}^{+}\right.$and $\left.\mathrm{Cl}^{-}\right)$fluxes have a mediator in common. On the right, checkboxes are generated for the visualized fluxes and channels in order to allow the user to move them across the membranes.

Direction of a flux is represented with a line, an arrow symbol, and a text to define the solute being transported. This direction is defined in the biological information deposited in PMR with source and sink 


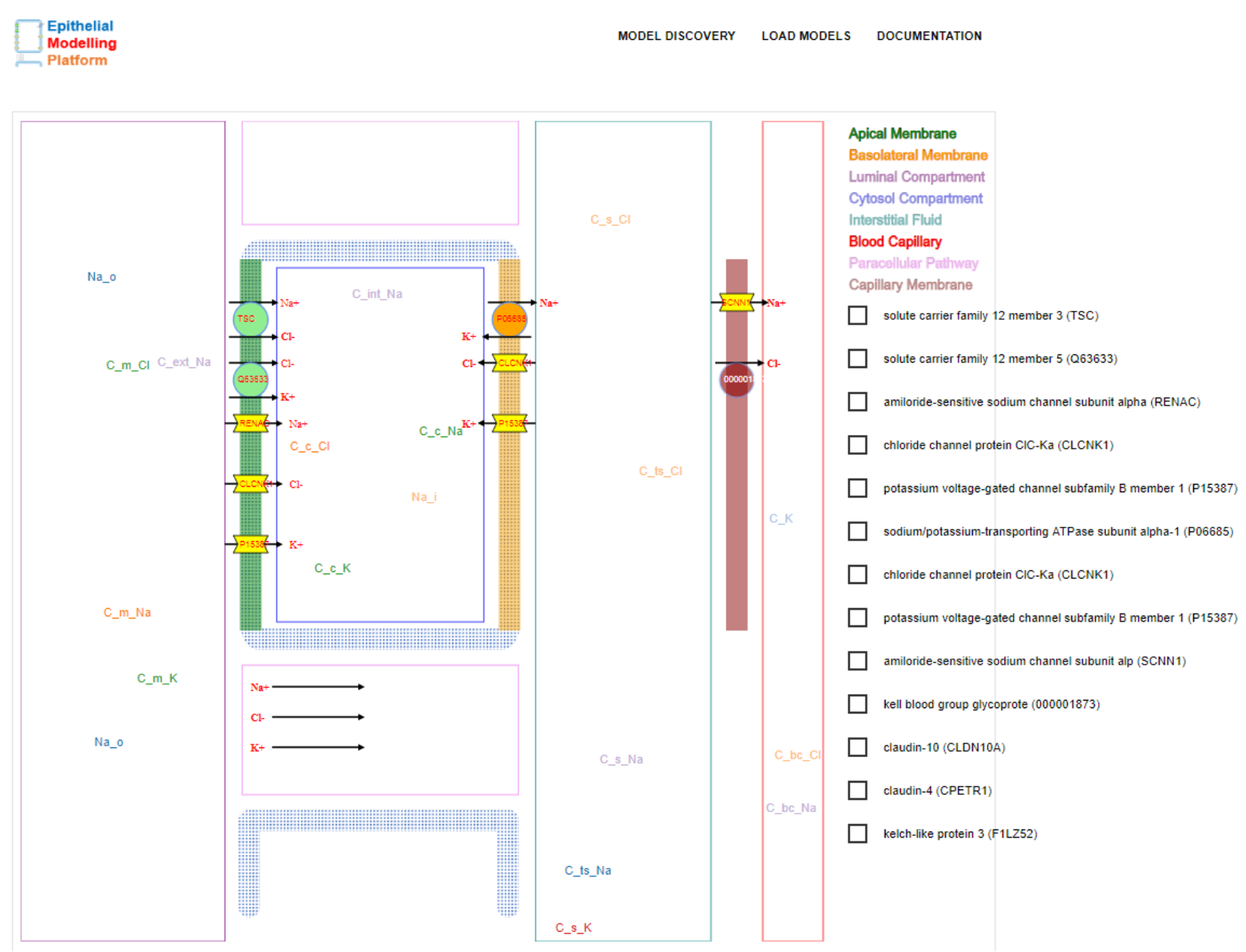

Figure 5. An example of the platform. It consists of five compartments and three membranes indicated by unique colors in the top-right corner. Physical entities and processes are represented with shapes such as: fluxes and co-transporters with circles; channels with polygons; and diffusive fluxes in the paracellular pathway with a text, a line, and an arrow. Arrows depict the direction of flow defined by the annotated biological semantics. On the right, checkboxes are generated for the visualized shapes (e.g. circles for fluxes, polygons for channels) on the apical, basolateral, and capillary membrane in order to allow the user to move across the membranes. Concentration of the annotated CellML variables float in the respective compartments. For example, $\mathrm{C} \_\mathrm{m} \_\mathrm{K}$ floats in the portion of renal filtrate in the distal convoluted tubule compartment and C_s_Na floats in the portion of tissue fluid in the epithelial cell of distal tubule compartment.

parameters. Here, source and sink parameters can be interchangeably either portion of renal filtrate in distal convoluted tubule, portion of cytosol in epithelial cell of distal tubule, or portion of tissue fluid in epithelial cell of distal tubule compartment. For instance, Fig. 5 illustrates that flux of sodium $\left(\mathrm{J} \_\mathrm{mc} \_\mathrm{Na}\right)$ and flux of chloride ( $\mathrm{J} / \mathrm{mc} \_\mathrm{Cl}$ ) flow from portion of renal filtrate in distal convoluted tubule to portion of cytosol in epithelial cell of distal tubule compartment across apical plasma membrane and TSC co-transporter. In contrast, concentration of annotated CellML variables float in the respective compartments. For example, $\mathrm{C} \_\mathrm{m} \_\mathrm{K}$ floats in the portion of renal filtrate in distal convoluted tubule compartment and $\mathrm{C} \_\mathrm{s} \_\mathrm{Na}$ in the portion of tissue fluid in epithelial cell of distal tubule compartment. 


\author{
Recommender System \\ sodium/hydrogen exchanger 3 is a Kidney model. It is located in Epithelial cell of \\ proximal tubule, Apical plasma membrane, Proximal convoluted tubule. \\ Model: weinstein_1995.cellml\#NHE3.J_NHE3_Na \\ 3 \\ Species: Rattus norvegicus \\ Gene: Slc9a3 \\ Protein: sodium/hydrogen exchanger 3 \\ Recommendations/suggestions based on existing models in PMR \\ Basolateral membrane model \\ $\square$ sodium/hydrogen exchanger 3 (human) \\ $\square$ sodium/potassium-transporting ATPase subunit alpha-1 (rat)
}

Biological Meaning: Flux of $\mathrm{Na}+$ from portion of renal filtrate in proximal convoluted tubule to portion of cytosol in epithelial cell of proximal tubule through sodium/hydrogen exchanger

Alternative model of sodium/hydrogen exchanger 3

Not Exist

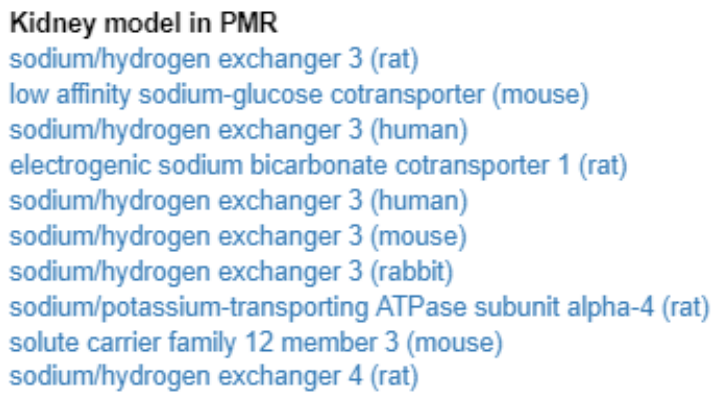

Kidney model in PMR

sodium/hydrogen exchanger 3 (rat)

low affinity sodium-glucose cotransporter (mouse)

sodium/hydrogen exchanger 3 (human)

electrogenic sodium bicarbonate cotransporter 1 (rat)

sodium/hydrogen exchanger 3 (human)

sodium/hydrogen exchanger 3 (mouse)

sodium/hydrogen exchanger 3 (rabbit)

sodium/potassium-transporting ATPase subunit alpha-4 (rat)

solute carrier family 12 member 3 (mouse)

sodium/hydrogen exchanger 4 (rat)

Figure 6. Recommender system as a pop-up window, with some information and suggestions, when a circle represented as "flux of $\mathrm{Na}^{+}$from portion of renal filtrate in distal convoluted tubule to portion of cytosol in epithelial cell of distal tubule through the sodium/hydrogen exchanger 3 (NHE3) (Weinstein. 1995) model" is moved from apical to basolateral membrane.

\title{
Recommender System
}

On the SVG-based modelling platform of the EMP, we have provided a feature to make recommendations or suggestions for the user to perform graphical editing. In this case, users can drag a model from apical to basolateral membrane or vice versa, then a pop-up window will appear with a brief desription of the dragged model extracted from the biological semantics in PMR, as well as some suggestions provided by the EBI web services. Here, we have applied our concept presented in (Sarwar et al., 2018) in regard to find similar models as suggestions.

Similar to Figure 4 in (Sarwar et al., 2018), a list of suggestions begins with a brief description of the dragged model: CellML model entity with an URI pointing to the PMR workspace, species and gene used during the experiment, and a protein name. Next, this recommender system provides some suggestions on existing annotated models as opposed to the dragged model's membrane. Fig. 6 shows an example when "flux of sodium from portion of renal filtrate in distal convoluted tubule to portion of cytosol in 
epithelial cell of distal tubule through the sodium/hydrogen exchanger 3 (NHE3) (Weinstein, 1995) model" is dragged from apical to basolateral membrane. Therefore, the recommender system extracts existing annotated models in the basolateral membrane from PMR. Users can choose one of the suggested models to replace with the dragged NHE3 model.

To populate the suggested models, we have leveraged two web services hosted at the EBI: WSDbfetch (WSDbfetch, 2019) and Clustal Omega (Clustal, 2019; Sievers et al., 2011). The WSDBfetch was used to get protein sequences for a list of protein identifiers. These protein sequences were formatted as JSON and then dispatched to the Clustal Omega web service to get a comparison score between the protein sequences in a representation of a similarity matrix. Fig. 7)illustrates a similarity matrix retrieved from the EBI Clustalo Omega web service.

On the pop-up window, another recommendation is made as an alternative model of the dragged NHE3 model, i.e. same protein but different species, genes, or workspaces. In this case, "Not Exist" shows in Fig. 6. Lastly, a list of related organ models is shown for the users to investigate further.

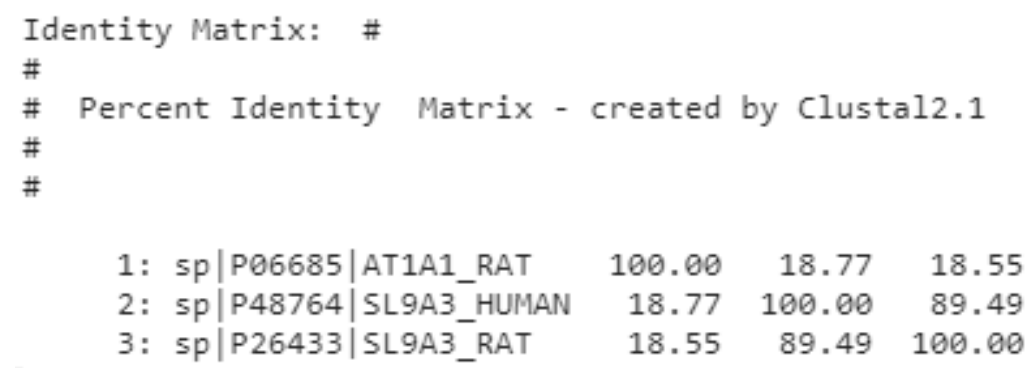

Figure 7. A similarity matrix retrieved from the EBI clustalo omega web service where P26433 is the dragged protein model and the remaining protein models are from Fig. 6, see list of proteins under "Basolateral membrane model" section header. Ranking of these proteins are based on IDs and matrix scores: P48764 (89.49) and P06685 (18.55), with respect to the dragged P26433 protein model (NHE3).

\section{Model Assembly Service}

The end result of our model discovery and assembly is a recipe that consists of models' components and relevant information to help construct a new epithelial model. To achieve this, we have implemented a model assembly service which does model composition by utilizing the libCellML (libCellML, 2019) library. The source code of the model assembly service is available at https://github.com/dewancse/ epithelial-modelling-platform/tree/master/server.

\section{libCelIML}

libCellML is a library to serialize, validate, and instantiate a CellML model by utilizing convenience methods and objects in $\mathrm{C}++$. It also provides Python bindings to access these convenience methods and objects in Python. The source code and documentation of libCellML are available at https://github. com/cellml/libcellml and http://libcellml.readthedocs.io/.

\section{Model-recipe and Composition}

The first step of the model assembly service was to iterate over the model entities in the model-recipe with a view to extracting models' components and then importing these components and the associated variables in the new model. Fig. 8 shows an example of eleven models' components which is reproduced from Fig. 5. For example, Na-Cl co-transporter (TSC) is located on the apical membrane where " $\mathrm{Na}^{+}$and 
$\mathrm{Cl}^{-}$flow from portion of renal filtrate in distal convoluted tubule ("Luminal Compartment" indicated in the top-right corner in Fig. 8) to portion of cytosol in epithelial cell of distal tubule ("Cytosol Compartment" indicated in the top-right corner in Fig. 8) compartment"; and Na-K pump (P06685) is located on the basolateral membrane where " $\mathrm{Na}^{+}$flows from portion of cytosol in epithelial cell of distal tubule to portion of tissue fluid in epithelial cell of distal tubule ("Interstitial Fluid" indicated in the top-right corner in Fig. 8) compartment" and " $\mathrm{K}^{+}$flows from portion of tissue fluid in epithelial cell of distal tubule to portion of cytosol in epithelial cell of distal tubule compartment". Relevant concentration of solutes float in the portion of renal filtrate in distal convoluted tubule, portion of cytosol in epithelial cell of distal tubule, and portion of tissue fluid in epithelial cell of distal tubule compartment.

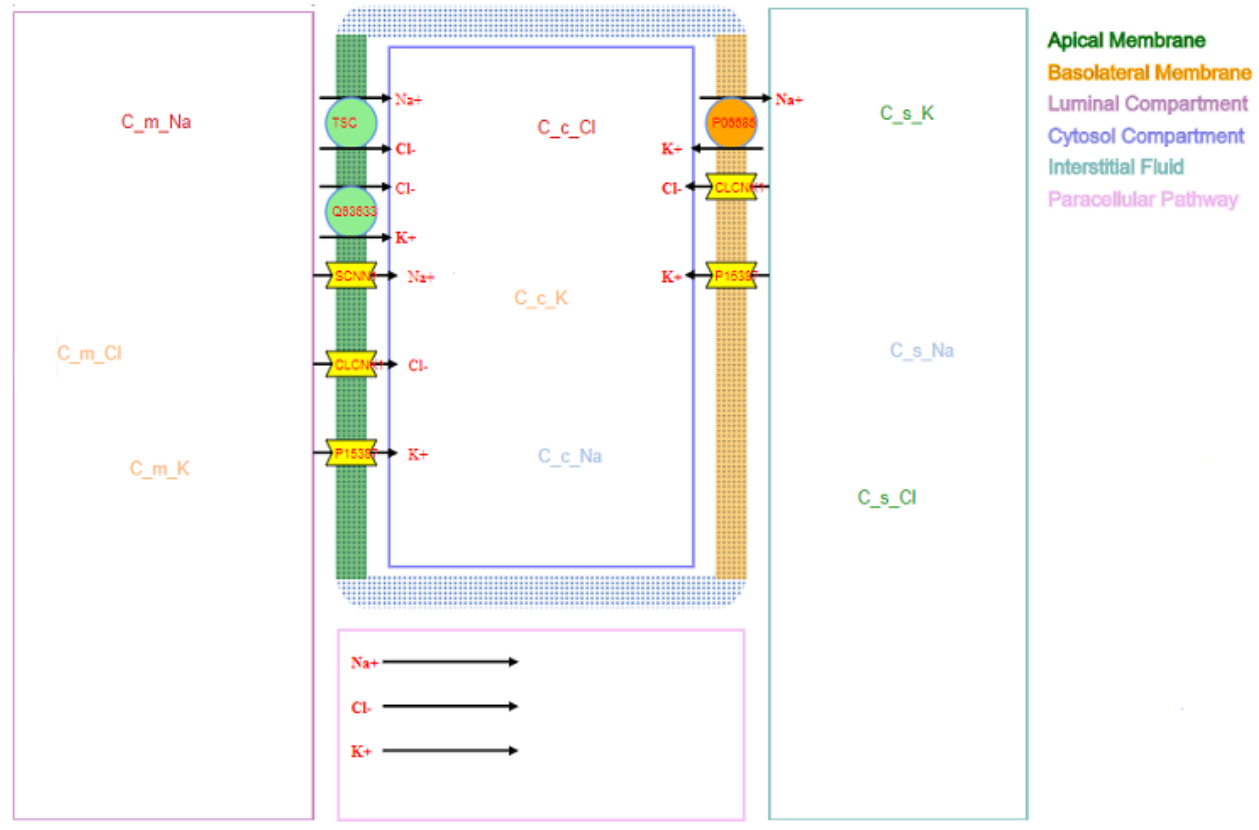

Figure 8. An example of the platform where $\mathrm{Na}-\mathrm{Cl}$ and $\mathrm{K}-\mathrm{Cl}$ co-transporters, $\mathrm{Na}$ channel, $\mathrm{K}$ channel, and $\mathrm{Cl}$ channel are located on the apical membrane; $\mathrm{Na}-\mathrm{K}$ pump, $\mathrm{K}$ channel, and $\mathrm{Cl}$ channel are located on the basolateral membrane; and $\mathrm{Na}, \mathrm{K}$ and $\mathrm{Cl}$ diffusive fluxes are located on the paracellular compartment. Respective concentration of solutes float in the portion of renal filtrate in distal convoluted tubule ("Luminal Compartment"), portion of cytosol in epithelial cell of distal tubule ("Cytosol Compartment") and portion of tissue fluid in epithelial cell of distal tubule ("Interstitial Fluid") compartment.

Fig. 9 shows a model-recipe, which is the background information of the visualized models in Fig. 8 . This recipe has a detailed annotated information to construct a new epithelial model. In Fig. 9, med fma denotes mediator FMA which has the URI of apical plasma membrane; med pr denotes solute carrier family 12 member 3 or thiazide-sensitive sodium-chloride co-transporter; med_pr_text and med_pr_text_syn denote the textual representation and synonym (in short form) of the med_pr, respectively; model_entity combines model name (chang_fujita_b_1999.cellml), component name (total_transepithelial_sodium_flux) and variable name $\left(\mathrm{J} \_\mathrm{mc} \_\mathrm{Na}\right)$; model_entity 2 is the second part of this co-transporter where model name is chang_fujita_b_1999.cellml, component name is solute_concentrations and variable name is J_mc_Cl; protein_name, in this case, is a reference URI of the epithelial cell.

As shown in Fig. 8, " $\mathrm{Na}^{+}$and $\mathrm{Cl}^{-}$flow from portion of renal filtrate in distal convoluted tubule to portion of cytosol in epithelial cell of distal tubule compartment across apical plasma membrane and $\mathrm{Na}-\mathrm{Cl}$ co-transporter (TSC)", thus, source_fma is a reference URI of portion of renal filtrate in distal convoluted 


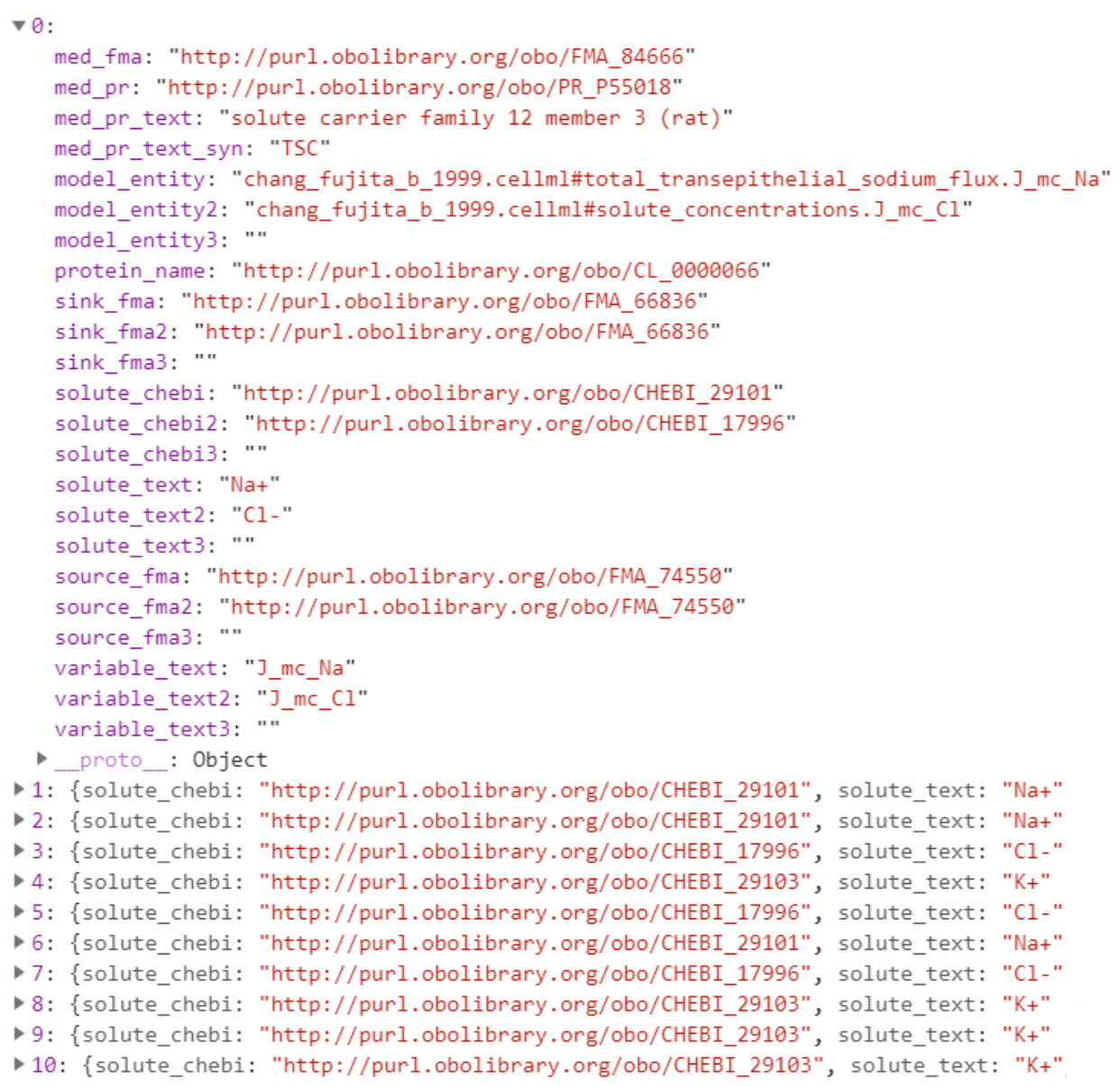

Figure 9. A detailed annotated information encapsulated inside a $\mathrm{Na}-\mathrm{Cl}$ co-transporter (TSC) object visualized on the apical membrane in Fig. 8, which is a recipe to perform model composition by utilizing the libCellML (libCellML, 2019). The remaining co-transporters and channels visualized in Fig. 8 are kept hidden for convenience.

tubule compartment, sink fma is a reference URI of portion of cytosol in epithelial cell of distal tubule compartment, solute_chebi is a reference URI of sodium, solute_text is a short form of sodium, variable_text is a CellML variable name of sodium, source fma2 is a reference URI of portion of renal filtrate in distal convoluted tubule compartment, sink fma2 is a reference URI of portion of cytosol in epithelial cell of distal tubule compartment, solute_chebi2 is a reference URI of chloride, solute_text2 is a short form of chloride, and variable_text2 is a CellML variable name of chloride. Similarly, Na-K pump in Fig. 8 has a detailed annotated information which is kept hidden in Fig. 9 for convenience.

In addition to importing components and the associated variables from the source models in the modelrecipe, we encapsulated the imported components inside an epithelial component. Next, we created an environment component that contains a time variable, and also created three components - lumen (portion of renal filtrate in distal convoluted tubule), cytosol (portion of cytosol in epithelial cell of distal tubule), and interstitial fluid (portion of tissue fluid in epithelial cell of distal tubule) to store relevant variables from the model-recipe. The epithelial component contains additional variables that are required in the lumen, cytosol, and interstitial fluid component.

Following step involves iterating over the model entities in the model-recipe and then extracting variable and component names. As mentioned above, we encapsulated the extracted components inside an epithelial 
component. On the other hand, variable names were used to represent fluxes, channels, and diffusive fluxes. We constructed ordinary differential equations (ODE) to represent fluxes. However, for channels and diffusive fluxes, we extracted the full mathematical equations from the source models in the model-recipe. To construct these equations, we instantiated a time variable in the lumen, cytosol, and interstitial fluid component.

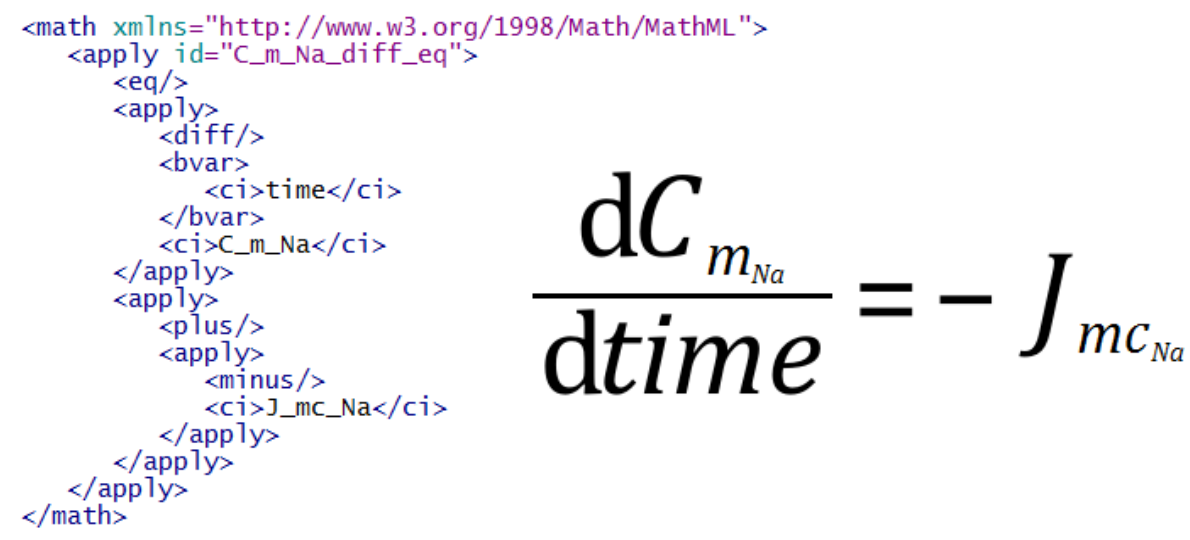

Figure 10. A MathML (MathML, 2019) representation of "flux of sodium" (J_mc $\_$a) which is an ordinary differential equation for "concentration of sodium" (C_m_Na) with respect to time.

To construct an ODE equation, we need to have concentration of solutes in the respective compartments - portion of renal filtrate in distal convoluted tubule, portion of cytosol in epithelial cell of distal tubule, or portion of tissue fluid in epithelial cell of distal tubule. For example, to construct an ODE equation for "flux of sodium from portion of renal filtrate in distal convoluted tubule to portion of cytosol in epithelial cell of distal tubule compartment across apical plasma membrane", we need to have "concentration of sodium in the portion of renal filtrate in distal convoluted tubule" and "concentration of sodium portion of cytosol in epithelial cell of distal tubule" compartment, then we can make an ODE equation which will measure "flux of sodium from portion of renal filtrate in distal convoluted tubule to portion of cytosol in epithelial cell of distal tubule" compartment, i.e. "sodium flows from portion of renal filtrate in distal convoluted tubule to portion of cytosol in epithelial cell of distal tubule" compartment. To get the respective concentration of solutes, we made a SPARQL call to PMR for a given solute (sodium, chloride, etc) and compartment (portion of renal filtrate in distal convoluted tubule, portion of cytosol in epithelial cell of distal tubule, or portion of tissue fluid in epithelial cell of distal tubule). The result of this call was a list of concentration of the specified solute in the specified compartment. Then we iterated over each concentration variable in the result and checked that whether both the flux and concentration variable reside in the same component. In the ODE equation, we marked with plus or minus sign notation to denote in which direction the concentration of the solute flows. After getting the respective concentration variable of the flux and the notation, we constructed an ODE equation by utilizing a MathML (MathML, 2019). Fig. 10 illustrates an ODE equation - concentration of sodium in the portion of renal filtrate in distal convoluted tubule compartment (C_m_Na) with respect to time is equal to negative flow of concentration of sodium ( $\mathrm{J} \_\mathrm{mc} \_\mathrm{Na}$ ). This flow is negative because concentration of sodium is moving from portion of renal filtrate in distal convoluted tubule to portion of cytosol in epithelial cell of distal tubule compartment.

In contrast, to construct mathematical equations for channels and diffusive fluxes, we extracted the full math equation from the source model in the model-recipe. For example, Fig. 11 shows an example equation of a sodium channel from the Chang and Fujita model (Chang and Fujita, 1999). We extracted 


$$
G_{m c_{N a}}=P_{m c_{N a}} \cdot \frac{F \cdot\left(\psi_{m}-\psi_{c}\right)}{R T} \cdot \frac{C_{m_{N a}}-C_{c_{N a}} \cdot e^{-\frac{F}{R T} \cdot\left(\psi_{m}-\psi_{c}\right)}}{1-e^{-\frac{F}{R T} \cdot\left(\psi_{m}-\psi_{c}\right)}}
$$

Figure 11. Equation of a "sodium channel" $\left(\mathrm{G} \_\mathrm{mc} \_\mathrm{Na}\right)$ visualized as polygons (SCNN1) on the apical membrane in Fig. 8 .

this channel's equation and traversed through the variables of this equation in order to instantiate them in the epithelial component of the new model.

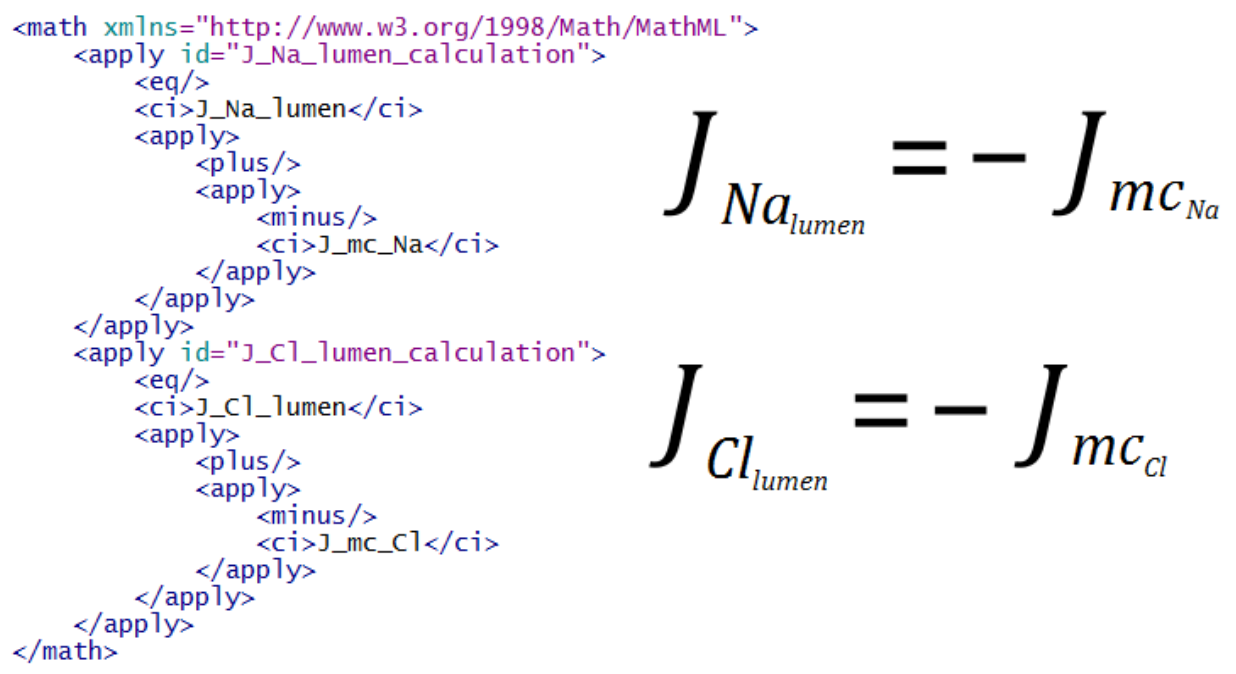

Figure 12. A MathML (MathML, 2019) representation of "total flux of sodium" (J_Na_lumen) and "total flux of chloride" (J_Cl_lumen) in the portion of renal filtrate in distal convoluted tubule (Luminal Compartment) compartment in Fig. 8 .

In terms of total fluxes, channels and diffusive fluxes equations, we evaluated out-going and in-coming fluxes of components and then constructed equations. For example, Fig. 12 illustrates "total flux of sodium" and "total flux of chloride" in the portion of renal filtrate in distal convoluted tubule (luminal compartment) compartment of Fig. 8, In the final step of the model assembly service, we mapped variables between the epithelial component and its encapsulated components. We also mapped variables between epithelial component's encapsulated siblings.

\section{DISCUSSION}

We have developed a novel web-based platform, the Epithelial Modelling Platform (EMP), for scientists to discover and explore mathematical models relevant to their work. On this platform, they can semantically visualize models for graphical editing and model assembly with a view to constructing a new model. In particular, this involves three steps: annotation of mathematical models with biological information; generation of metadata using the SemGen tool; and the EMP to discover models from the metadata in order to visualize models for graphical editing and model assembly and construction.

To perform biological annotation, we have comprehensively annotated a cohort of epithelial transport models: twelve renal and four lung models, which are available at https://models. physiomeproject.org/workspace/527. In particular, we pulled out mathematical terms from 
each model and annotated with biological information. In addition to this, we curated the models with protein identifiers from the UniProt database, species and gene names used during the experiments of the models, compartment names, and anatomical locations. Next, we fed these biological information onto the SemGen tool to generate metadata. This metadata is then deposited in PMR which are discoverable, accessible and persistent.

To discover and explore the epithelial transport models, we have developed the EMP that utilizes cascaded SPARQL calls to iteratively retrieve information from PMR. First, we make a query with a protein identifier to the OLS in order to get a human-readable name. Subsequent call retrieves protein's species and gene name. In addition to model discovery, users are able to add protein models and generate metadata in PMR. The EMP allows users to semantically display a collection of discovered models for graphical editing and model assembly. This "graphical editing" has been integrated with a recommender system where users can rank similar models based on the suggestions provided by the EBI web services.

The end result of the graphical editing and model assembly is the generation of a model-recipe, which is a collection of components from disparate discovered models. Fig. 9 illustrates an example model-recipe that has the required information to perform model composition. This recipe is then dispatched to our model assembly service in order to compose a new epithelial model. To perform model composition, we have utilized convenience methods and objects of the libCellML library.

The integration and discovery of the semantic annotation visualized on the SVG-based epithelial platform is a novel application of the services provided by PMR that would inspire users to reuse the data and models. Integration of data and models with the provision of semantic web technologies will integrate computational model of various organ systems, which is the ultimate effort of the Physiome Project and the VPH. We believe this approach with the aid of modern tools and technologies would provide an excellent epithelial modelling platform. Novice modellers could use this platform as a learning tool.

The "Add Model" feature enables scientists to create a new CellML model and metadata. In the future, we would like to extend this feature and implement a notification system between CellML editorial board and the scientists so that the CellML editorial board can incorporate mathematical equations and validate the new CellML model.

\section{CONFLICT OF INTEREST STATEMENT}

The authors declare that the research was conducted in the absence of any commercial or financial relationships that could be construed as a potential conflict of interest.

\section{FUNDING}

DMS was supported by the Medical Technologies Centre of Research Excellence's Doctoral Scholarship. DPN was supported by an Aotearoa Foundation Fellowship. JHG, BEC, and MLN were supported by the National Institutes of Health grant R01LM011969.

\section{ACKNOWLEDGMENTS}

The authors wish to acknowledge the Centre for eResearch at the University of Auckland for their help in facilitating this research. The availability of our live web application demonstration is made possible by use of the Nectar Research Cloud, a collaborative Australian research platform supported by the 
National Collaborative Research Infrastructure Strategy (NCRIS). Thanks to Sean Matheny at the Center for eResearch for his cooperation to setup our tool and service in Center for eResearch at the University of Auckland and Nectar.

\section{AVAILABILITY OF DATA AND MATERIALS}

The source code and links to the live demonstration of the Epithelial Modelling Platform are available at https://github.com/dewancse/epithelial-modelling-platform.

\section{REFERENCES}

Chang, H. and Fujita, T. (1999). A numerical model of the renal distal tubule. American Journal of Physiology 276, F931-F951. doi:10.1152/ajprenal.1999.276.6.F931

Clustal (2019). EBI webservice to get multiple sequence alignment. https:/ /www.ebi .ac.uk/ seqdb/confluence/display/WEBSERVICES/clustalo\$_\$rest. Accessed 21 January.

COMBINE (2019). The COmputational Modeling in Biology NEtwork (combine). http: / / co. mbine. org/. Accessed 21 January.

Cook, D. L., Neal, M. L., Hoehndorf, R., Gkoutos, G. V., and Gennari, J. H. (2013). Representing physiological processes and their participants with PhysioMaps. Biomedical Semantics 4. doi:10.1186/ 2041-1480-4-S1-S2

Cook, D. L., Wiley, J. C., and Gennari, J. H. (2007). Chalkboard: ontology-based pathway modelling and qualitative inference of disease mechanisms. Pacific Symposium on Biocomputing , 16-27

Cooper, J., Cervenansky, F., De Fabritiis, G., Fenner, J., Friboulet, D., Giorgino, T., et al. (2010). The Virtual Physiological Human toolkit. Philosophical Transactions of the Royal Society of London A: Mathematical, Physical and Engineering Sciences 368, 3925-3936. doi:10.1098/rsta.2010.0144

Cooper, J., Scharm, M., and Mirams, G. R. (2015a). The Cardiac Electrophysiology Web Lab. PeerJ PrePrints doi:10.7287/peerj.preprints.1338v1

Cooper, J., Vik, J. O., and Waltemath, D. (2015b). A call for virtual experiments: Accelerating the scientific process. Progress in Biophysics and Moldecular Biology 117, 99-106. doi:10.1016/j.pbiomolbio.2014. 10.001

Coskun, S. A., Cicek, A. E., Lai, N., Dash, R. K., Ozsoyoglu, Z. M., and Ozsoyoglu, G. (2013). An online model composition tool for system biology models. BMC Systems Biology 7, 88. doi:10.1186/ 1752-0509-7-88

Courtot, M., Juty, N., Knüpfer, C., Waltemath, D., Zhukova, A., Dräger, A., et al. (2011). Controlled vocabularies and semantics in systems biology. Molecular Systems Biology 7. doi:10.1038/msb.2011.77

Cuellar, A., C.M., L., P.F., N., D.P., B., Nickerson, D., and P.J., H. (2003). An overview of CellML 1.1, a biological model description language. SIMULATION 79, 740-747. doi:10.1177/0037549703040939

de Boer, T. P., van der Werf, S., Hennekam, B., Nickerson, D. P., Garny, A., Gerbrands, M., et al. (2017). esolv, a cellml-based simulation front-end for online teaching. Advances in Physiology Education 41, 425-427. doi:10.1152/advan.00127.2016

de Bono, B., Grenon, P., Helvensteijn, M., Kok, J., and Kokash, N. (2014). ApiNATOMY: Towards multiscale views of human anatomy. The 13th International Symposium on Intelligent Data Analysis (IDA) 8819. doi:10.1007/978-3-319-12571-8_7

de Bono, B., Helvensteijn, M., Kokash, N., Martorelli, I., Sarwar, D., Islam, S., et al. (2016). Requirements for the formal representation of pathophysiology mechanisms by clinicians. Interface Focus Journal 6 , 20150099. doi:10.1098/rsfs.2015.0099 


\section{Sarwar et al.}

Galdzicki, M., Rodriguez, C., Chandran, D., Sauro, H. M., and Gennari, J. H. (2011). Standard biological parts knowledgebase. PLOS ONE 6, e17005. doi:10.1371/journal.pone.0017005

Garny, A., Cooper, J., and Hunter, P. J. (2010). Toward a VPH/Physiome toolkit. Wiley Interdisciplinary Reviews: Systems Biology and Medicine 2, 134-147. doi:10.1002/wsbm.63

Garny, A. and Hunter, P. J. (2015). Opencor: a modular and interoperable approach to computational biology. Frontiers in Physiology 6. doi:10.3389/fphys.2015.00026

Gennari, J. H., Neal, M. L., Carlson, B. E., and Cook, D. L. (2008). Integration of Multi-scale Biosimulation Models via Light-weight Semantics. Pacific Symposium on Biocomputing , 414-425

Gennari, J. H., Neal, M. L., Galdzicki, M., and Cook, D. L. (2011). Multiple ontologies in action: Composite annotations for biosimulation models. Journal of Biomedical Informatics 44, 146-154. doi:10.1016/j.jbi.2010.06.007

Hoehndorf, R., Dumontier, M., Gennari, J. H., Wimalaratne, S., de Bono, B., Cook, D. L., et al. (2011). Integrating systems biology models and biomedical ontologies. BMC Systems Biology 5, 124. doi:10. 1186/1752-0509-5-124

Hucka, M., Finney, A., Sauro, H. M., Bolouri, H., Doyle, J. C., Kitano, H., et al. (2003). The systems biology markup language (sbml): a medium for representation and exchange of biochemical network models. Bioinformatics Oxford Journals 19, 524-531

Hunter, P., Coveney, P. V., de Bono, B., Diaz, V., Fenner, J., Frangi, A. F., et al. (2010). A vision and strategy for the virtual physiological human in 2010 and beyond. Philos Transact A Math Phys Eng Sci 368, 2595-614. doi:10.1098/rsta.2010.0048

Hunter, P. J. and Borg, T. K. (2003). Integration from proteins to organs: the Physiome Project. Nature Reviews Molecular Cell Biology 4, 237-243. doi:10.1038/nrm1054

Krause, F., Uhlendorf, J., Lubitz, T., Schulz, M., Klipp, E., and Liebermeister, W. (2010). Annotation and merging of sbml models with semanticsbml. Bioinformatics 26, 421-422. doi:10.1093/bioinformatics/ btp642

libCellML (2019). libCellML. https://libcellml.readthedocs.io/en/latest/index. html. Accessed 21 January.

Lister, A. L., Pocock, M., Taschuk, M., and Wipat, A. (2009). Saint: a lightweight integration environment for model annotation. Oxford Bioinformatics 25, 3026-3027. doi:10.1093/bioinformatics/btp523

Mackenzie, M., Loo, D., Panayotova-Heiermann, M., and Wright, E. M. (1996). Biophysical characteristics of the pig kidney na/glucose cotransporter sglt2 reveal a common mechanism for sglt1 and sglt2. Journal of Biological Chemistry 271, 32678-32683. doi:10.1074/jbc.271.51.32678

MathML (2019). Mathematical Markup Language (mathml). https : / www .w3 . org/TR/MathML3/. Accessed 21 January.

Neal, M. L., Carlson, B. E., Thompson, C. T., James, R. C., Kim, K. G., Tran, K., et al. (2015). Semanticsbased composition of integrated cardiomyocyte models motivated by real-world use cases. PLoS ONE 10, e0145621. doi:10.1371/journal.pone.0145621

Neal, M. L., Cook, D. L., and Gennari, J. H. (2013). An owl knowledge base for classifying and querying collections of physiological models: A prototype human physiome. Proceedings of the 2013 International Conference on Biomedical Ontology.

Neal, M. L., Gennari, J. H., Arts, T., and Cook, D. L. (2009). Advances in Semantic Representation for Multiscale Biosimulation: A Case Study in Merging Models. Pacific Symposium on Biocomputing, $304-315$ 
Neal, M. L., Thompson, C. T., Kim, K. G., James, R. C., Cook, D. L., Carlson, B. E., et al. (2018). Semgen: a tool for semantics-based annotation and composition of biosimulation models. Bioinformatics , bty829doi:10.1093/bioinformatics/bty829

OLS (2019). EBI Ontology Lookup Service. https://www.ebi.ac.uk/ols/index. Accessed 21 January.

Piñero, J., Queralt-Rosinach, N., Àlex Bravo, Deu-Pons, J., Bauer-Mehren, A., Baron, M., et al. (2015). DisGeNET: a discovery platform for the dynamical exploration of human diseases and their genes. Database (Oxford) 2015, bav028. doi:10.1093/database/bav028

Sarwar, D. M., Kalbasi, R., Gennari, J. H., Carlson, B. E., Neal, M. L., de Bono, B., et al. (2018). Model annotation and discovery with the physiome model repository. bioRxiv doi:10.1101/498501

Sievers, F., Wilm, A., Dineen, D., Gibson, T. J., Karplus, K., Li, W., et al. (2011). Fast, scalable generation of high-quality protein multiple sequence alignments using clustal omega. Molecular Systems Biology 7. doi:10.1038/msb.2011.75

Weinstein, A. (1995). A kinetically defined $\mathrm{Na}+\mathrm{H}+$ antiporter within a mathematical model of the rat proximal tubule. The Journal of General Physiology 105, 617-641

Wilkinson, M. D. and et al. (2016). The FAIR Guiding Principles for scientific data management and stewardship. Scientific Data 3. doi:10.1038/sdata.2016.18

Wimalaratne, S. M., Halstead, M. D. B., Lloyd, C. M., Cooling, M. T., Crampin, E. J., and Nielsen, P. F. (2004). Web-based kinetic modelling using JWS Online. Applications Note in Bioinformatics Oxford Journal 20, 2143-2144. doi:10.1093/bioinformatics/bth200

WSDbfetch (2019). EBI webservice to get entries from various biological databases. https:// WwW.ebi.ac.uk/seqdb/confluence/pages/viewpage.action?pageId=48923608. Accessed 21 January.

Yu, T., Lloyd, C. M., Nickerson1, D. P., Cooling, M. T., Miller, A. K., Garny, A., et al. (2011). The Physiome Model Repository 2. Bioinformatics Oxford Journals 27, 743-744. doi:10.1093/bioinformatics/btq723 